\section{Tratamiento del síndrome del túnel del carpo}

Carpal tunnel syndrome treatment

\section{Félix Omar López Contreras}

Especialista en Traumatología y

Ortopedia. Hospital General del Norte

de Guayaquil Los Ceibos,

dromarlopezc@gmail.com.

https://orcid.org/0000-0003-1917-

4484, Guayaquil, Ecuador

Xavier Palomeque Salazar

Especialista en Traumatología y

Ortopedia. Hospital General del Norte

de Guayaquil Los Ceibos,

xavierpalomeque@gmail.com,

https://orcid.org/0000-0002-3603-

0442, Guayaquil, Ecuador

Frank Marino Rojas Alvarado

Médico de posgrado. Hospital

General del Norte de Guayaquil Los

Ceibos, eljaico@hotmail.com,

https://orcid.org/0000-0002-1304-

4833, Guayaquil, Ecuador

Eulixis Cornelio Estupiñan Gómez

Médico general. Hospital General del

Norte de Guayaquil Los Ceibos,

eulises_esgo@hotmail.com,

https://orcid.org/0000-0003-3882-

6396, Guayaquil, Ecuador

http://www.jah-journal.com/index.php/jah

Journal of American health

Julio - Diciembre vol. 3. Num. 2 - 2020

Esta obra está bajo una Licencia Creative Commons

Atribución-NoComercial-CompartirIgual 4.0 Internacional.

RECIBIDO: 17 DE MARZO 2019

ACEPTADO: 13 DE AGOSTO 2019

PUBLICADO: 2 DE JULIO 2020

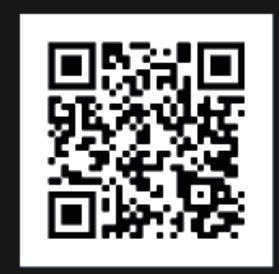

Scan this QR

code with your

smart phone or

mobile device to

read more papers

\section{RESUMEN}

Esta revisión narrativa analiza la literatura para determinar los conceptos actuales sobre el tratamiento de síndrome del túnel carpiano que permitan actualizar los conocimientos al personal sanitario. Se utilizó repositorios digitales como Pubmed, Elsevier y SCIELO para la búsqueda avanzada de la bibliografía sobre el tema, usando las palabras clave específicas "túnel carpiano", "tratamiento", "parestesias" y "electromiografía". Se analizaron artículos con menos de cinco años de publicación que resultaron de la búsqueda avanzada de la información. Conclusión: Esta revisión aclara algunos aspectos sobre el tratamiento de esta enfermedad. Las opciones de tratamiento conservador incluyen inmovilizar la muñeca en una posición neutral y fisioterapia. Los corticosteroides orales pueden ser efectivos a corto plazo (dos a cuatro semanas), pero las inyecciones locales pueden mejorar los síntomas durante un período más prolongado. Si los síntomas son refractarios a las medidas conservadoras o si los estudios de conducción nerviosa muestran un atrapamiento severo, puede ser necesaria la liberación abierta o endoscópico del túnel carpiano.

PALABRAS CLAVE: túnel carpiano, tratamiento, parestesias, electromiografía.

\section{ABSTRACT}

This narrative review analyzes the literature to determine the current concepts on the treatment of carpal tunnel syndrome that allow updating the knowledge of health personnel. Digital repositories such as Pubmed, Elsevier and SCIELO were used for 
the advanced search of the literature on the subject, using the specific keywords "carpal tunnel", "treatment", "paresthesia" and "electromyography". Articles with less than five years of publication that resulted from the advanced search for information were analyzed. Conclusion: This review clarifies some aspects of the treatment of this disease. Conservative treatment options include immobilizing the wrist in a neutral position and physical therapy. Oral corticosteroids can be effective in the short term (two to four weeks), but local injections can improve symptoms over a longer period. If symptoms are refractory to conservative measures or if nerve conduction studies show severe entrapment, open or endoscopic release of the carpal tunnel may be necessary.

KEYWORDS: carpal tunnel, treatment, paresthesias, electromyography.

\section{INTRODUCCIÓN}

El síndrome del túnel carpiano (STC) es una neuropatía por atrapamiento que implica la compresión del nervio mediano en la muñeca y se considera la más común de todas las mononeuropatías de atrapamiento focal (1). Representa el $90 \%$ de todas las neuropatías por atrapamiento diagnosticadas en países de gran desarrollo tecnológico y afecta a millones de personas en el mundo (2). Es causado por la compresión del nervio mediano a medida que viaja a través del túnel carpiano. Clásicamente, los pacientes con la afección experimentan dolor y parestesias en la distribución del nervio mediano, que incluye la cara palmar del pulgar, los dedos índice y medio, y la mitad radial del dedo anular (3). Las pistas adicionales incluyen hallazgos positivos del examen físico, como el signo de movimiento, maniobra de Phalen y la prueba de compresión del nervio mediano. Aunque los pacientes con síntomas y signos típicos del síndrome del túnel carpiano no necesitan pruebas adicionales, los estudios de ultrasonografía y electrodiagnóstico son útiles para confirmar el diagnóstico en casos atípicos y descartar otras causas (4).

El síndrome del túnel carpiano no es mortal, pero puede conducir a un daño completo e irreversible del nervio mediano, con la consiguiente pérdida severa de la función de la mano, si no se trata $(1,2)$. La incidencia del síndrome del túnel carpiano es de 1-3 casos por 1000 sujetos por año; La prevalencia es de aproximadamente 50 casos por 1000 sujetos en la población general. La incidencia puede aumentar hasta 150 casos por 1000 sujetos por año, con tasas de prevalencia superiores a 500 casos por 1000 sujetos en ciertos grupos de alto riesgo (4). La proporción entre mujeres y hombres para el síndrome del túnel carpiano es de 310: 1. El rango de edad pico para el desarrollo del STC es de 45 a 60 años, solo el $10 \%$ de los pacientes son menores de 31 años (5).

Actualmente se considera una complicación una patología que genera bastante incapacidad, obliga a la consulta por el servicio de urgencias, puede ser origen de complicaciones médicas potencialmente graves y tener incluso implicaciones laborales. Conocer los factores de riesgo es importante, ya que permitiría identificar pacientes con alta probabilidad de desarrollar la 
enfermedad y por ende se podrían utilizar tratamientos profilácticos. El presente artículo resume los conceptos actuales respecto al tratamiento del síndrome del túnel carpiano.

\section{MATERIALES Y MÉTODOS}

Se realizó una búsqueda de literatura médica para conocer las características generales del tratamiento del síndrome del túnel del carpo en bases de datos como PubMed, Elsevier y Scielo. La búsqueda incluyó artículos científicos desde enero del 2015 hasta diciembre del 2019. Se utilizaron estrategias de búsqueda con los siguientes términos del vocabulario MeSH: "túnel carpiano", "tratamiento", "parestesias", "electromiografía" y del vocabulario DeCS: síndrome del túnel del carpo, técnica percutánea, laserterpia, electrodiagnóstico. Se aplicaron los siguientes criterios de inclusión: artículos en idioma inglés y español, publicados en los últimos 5 años y realizados en humanos. El criterio de exclusión fue artículos cuyo contenido era sobre la epidemiología y fisiopatología del síndrome del túnel carpiano. Para lectura crítica en texto completo se escogieron 12 artículos y se adicionaron 5 artículos más procedentes de las citaciones de los documentos iniciales, los cuales se consideraron relevantes para la revisión de tema.

\section{RESULTADOS}

Los tratamientos para el STC son variables y dependen de la gravedad del síndrome. Clásicamente, el STC sin déficit neurológico se ha manejado de manera conservadora con inmovilización o inyecciones. Cuando el CTS sin déficit es resistente al tratamiento, es necesario un tratamiento más agresivo. De manera similar, cuando el STC se presenta con un déficit o en una forma aguda severa, puede buscarse un tratamiento quirúrgico $(1,4,5)$.

\section{Tratamiento conservador}

El tratamiento conservador del STC generalmente se considera terapia de primera línea a menos que un paciente presente un déficit sensorial o motor grave. El manejo conservador puede abarcar cualquiera o todas las siguientes intervenciones. Primero, la inmovilización de la muñeca a través de una férula rígida es la terapia inicial más común y evita el movimiento extremo al forzar la muñeca a una posición neutral con el objetivo de disminuir el edema y la fricción nerviosa (5).

Con menos frecuencia, se usa un aparato ortopédico más suave y flexible. La compensación por más movimiento es un mayor riesgo de edema y compresión. En coordinación con estas terapias, los suplementos orales han demostrado ser prometedores en estudios de casos. Para ayudar aún más a reducir el edema, los diuréticos orales tienen una eficacia marginal (1). La vitamina B6 es una coenzima en la función neural y tiene el potencial de ayudar a los pacientes con neuropatía no diagnosticada. Los esteroides orales y los AINE pueden ayudar a aliviar los síntomas, pero no a tratar el STC subyacente. Por último, se ha planteado la hipótesis de ciertos ejercicios físicos para reducir la presión y mejorar el retorno venoso al aumentar el deslizamiento del tendón y el nervio mediano $(4,5)$. Han demostrado tener un efecto terapéutico marginal paciente por paciente y deben considerarse en función de las preferencias de cada paciente.

En una gran revisión sistemática, los rasgos múltiples se asociaron en gran medida con la falta de respuesta al tratamiento conservador y pidieron que tanto el paciente como el médico 
reconsideraran la estrategia de tratamiento y consideraran vías más agresivas. Estos incluyeron la duración de los síntomas, la emaciación posterior y una prueba de Phalen positiva (6).

\section{Tratamiento quirúrgico}

En una gran revisión sistemática que incluyó más de cien estudios, las intervenciones quirúrgicas se compararon con el tratamiento conservador y las inyecciones de corticosteroides (7). Si bien los corticosteroides generalmente proporcionan un alivio más inmediato que los procedimientos quirúrgicos o las intervenciones conservadoras, el beneficio de las inyecciones disminuye con el tiempo; El alivio quirúrgico proporciona los resultados más efectivos a mediano y largo plazo. El procedimiento implica una incisión en el ligamento transverso del carpo para agrandar el túnel carpiano. La cirugía dura aproximadamente 10 minutos $y$, con aproximadamente 600,000 completados por año, se considera un procedimiento común en el campo de la cirugía (8).

Anteriormente, la cirugía se realizaba completamente abierta, pero ahora se lleva a cabo utilizando un abordaje mini-abierto o endoscópico. La técnica mini-abierta corta el techo del túnel carpiano, mientras que la técnica endoscópica es accionada por cámara y aumenta el tamaño del túnel internamente (6). Dada la simplicidad técnica del procedimiento, los esfuerzos para reducir los costos han comenzado a transferir la cirugía fuera de la sala de operaciones a una sala de procedimientos con esterilidad de campo (8). Los estudios han demostrado que esta transferencia está asociada con una reducción masiva en el costo sin comprometer las tasas de infección, las puntuaciones de satisfacción del paciente o el control del dolor postoperatorio.

El procedimiento para liberar la compresión conlleva un riesgo inherente y un potencial de complicaciones. El túnel carpiano contiene cuatro tendones FDP y cuatro tendones FDS, así como el tendón FPL y el nervio mediano. El daño de cualquiera de estos componentes durante la cirugía puede conducir a déficits motores o sensoriales (7). La lesión del nervio mediano, la estructura más superficial y más dañada en el túnel carpiano, puede ocurrir cuando se realiza la incisión en el ligamento transverso del carpo. Rara vez se dañan otras estructuras circundantes, como la rama tenar, los nervios digitales comunes y el nervio cubital, pero también pueden conducir a déficits motores y sensoriales (8). Además, si se cortan los vasos circundantes, como la arteria cubital y el arco palmar superficial, el campo se acumulará con sangre.

Estos vasos deben repararse para evitar lesiones isquémicas. Después de la operación, los problemas incluyen infección del sitio de la incisión, dolor y problemas en los tendones, así como debilidad bajo presión o durante los movimientos de agarre (6). La mayoría de estas complicaciones están asociadas con el tejido cicatricial y se resuelven espontáneamente en unos pocos meses. Si bien muchos pacientes sienten alivio inmediato, los síntomas de algunos pacientes pueden persistir. El fracaso de la liberación inicial del túnel carpiano es entre 7 y $25 \%$ (7); Las complicaciones son tan graves que del 5 al 12\% de los pacientes requieren una cirugía de revisión adicional. Estas revisiones pueden ser necesarias debido a una liberación incompleta, la necesidad de una compresión adicional o un diagnóstico incorrecto. En pacientes que tienen sistemas persistentes, se pueden inyectar corticosteroides para proporcionar alivio a corto plazo (8). La respuesta a estos corticosteroides tam bién es un buen predictor de si la cirugía posterior proporcionaría o no alguna utilidad. 


\section{Procedimiento mínimamente invasivo}

\section{Liberación endoscópica}

La liberación de compresión endoscópica se originó en 1989. Desde entonces se han presentado múltiples enfoques endoscópicos para el tratamiento de STC, pero con el tiempo, se ha demostrado que el enfoque que lleva el nombre de Agee et al, se asocia con los resultados más favorables. Se realiza una única incisión de $0.5-1.0 \mathrm{~cm}$ proximal al pliegue de flexión de la muñeca que sigue la superficie profunda del retináculo flexor hasta la almohadilla grasa distal. El seccionamiento del retináculo flexor se inicia distalmente. El riesgo de daño a los haces neurovasculares circundantes es inferior al 1\%, y el procedimiento se realiza en coordinación con un radiólogo como una liberación guiada por ultrasonido $(1,2)$.

Desafortunadamente, la confirmación de una cirugía exitosa no es posible a través del ligamento palmar del carpo porque no está bajo presión y, por lo tanto, no se separa una vez que se completa la liberación. Una revisión sistemática encontró que la liberación endoscópica es igualmente segura para un procedimiento abierto, no conlleva mayores riesgos y al mismo tiempo se asocia con menos tiempo libre del trabajo y retiro de las actividades diarias (9). El mismo grupo demostró en una revisión sistemática posterior de más de 2500 procedimientos que la liberación endoscópica fue tan efectiva para aliviar los síntomas y restaurar total o parcialmente el estado funcional como la técnica abierta.

\section{Terapia de inyección}

\section{Corticosteroides}

La inyección de corticosteroides se basa en los efectos antiinflamatorios de los esteroides para ayudar a aliviar los síntomas del síndrome del túnel carpiano. Se ha demostrado que las inyecciones de corticosteroides tienen reducciones similares en la puntuación del cuestionario del túnel carpiano de Boston (CTCB) en comparación con las férulas (2). Las inyecciones también se asocian con puntajes subjetivos más altos de satisfacción del paciente y cantidades disminuidas de analgésicos administrados que el grupo de férulas (10). Además, al comparar una combinación de los dos tratamientos conservadores contra las inyecciones solas, los individuos asignados al azar al grupo combinado mostraron mejoras aún mayores según lo determinado por el CTCB, así como una función nerviosa mejorada y una reducción de los síntomas. Por lo general, combinado con un anestésico local, el objetivo de la inyección es la cresta flexora de la muñeca en el lado cubital del tendón palmar. En los últimos años y con la mejora del ultrasonido, en lugar de usar puntos de referencia anatómicos, se ha demostrado que el uso de inyección guiada por ultrasonido conduce a una reducción significativa de los síntomas 1 mes después de la operación (11).

\section{Plasma rico en plaquetas}

Alternativamente, las inyecciones de plasma rico en plaquetas (PRP) contienen plaquetas concentradas y varios factores de crecimiento que pueden ayudar a aliviar los síntomas. Los mecanismos incluyen crecimiento, angiogénesis y regeneración de axones que podrían promover la regeneración del nervio mediano (12). La PRP ha demostrado su eficacia en una variedad de aplicaciones, desde odontología hasta ortopedia y desde neurocirugía hasta cosméticos (13). 


\section{Terapias alternativas}

\section{Terapia con láser}

El objetivo de la terapia con láser de bajo nivel es transferir energía a través de un haz para reducir la respuesta inflamatoria en un tejido objetivo. Mediante la inducción de la producción de endorfinas, serotonina y otros mediadores, el objetivo es reducir el edema e incluso promover la regeneración axonal (13).

\section{Terapia de ondas de choque}

La terapia de ondas de choque extracorpóreas es un tratamiento relativamente nuevo que se ha utilizado desde el cambio de siglo para los trastornos musculoesqueléticos inflamatorios y las neuropatías periféricas. Las ondas minishock inducen la regeneración axonal a través de varias vías y también han demostrado su eficacia en la reducción del dolor neuropático crónico. Las ondas mismas pueden administrarse en un patrón focalizado o radial. Las ondas radiales tienen la ventaja de dispersarse por toda un área, aunque a menor magnitud; lo que significa que no requieren la localización exacta del atrapamiento (14). En los estudios iniciales, la terapia de ondas de choque radiales se administró sin un efecto significativo en comparación con el grupo de control que acaba de recibir una férula.

\section{DISCUSIÓN}

Los tratamientos generalmente se agrupan en conservadores versus tratamientos quirúrgicos. Los 2 enfoques que han resultado en la satisfacción más consistente son la inyección de esteroides y la liberación del túnel carpiano (LTC). Las investigaciones disponibles sobre los resultados del tratamiento favorecen la inyección de esteroides y la cirugía (15). Las férulas utilizadas como tratamiento complementario parecen mejorar el dolor en muchos pacientes a pesar de no tener evidencia estadística significativa en muchos estudios. Además, de la reducción del dolor y la mejora del sueño, el uso de férulas ayuda a algunos pacientes a ser más ergonómicamente conscientes del uso de sus manos mientras deciden el tratamiento en función de sus necesidades individuales o esperan ser atendidos por un especialista en manos (16). Algunos pacientes afectados por STC prefieren retrasar la cirugía o evitarla por completo. El tratamiento más nuevo, la hidrodisección o la liberación del ligamento transverso del carpo bajo la guía de ultrasonido, se muestra prometedor con evidencia de investigación aún por llegar.

Los estudios han demostrado que parece haber una ventana para resultados óptimos de tratamiento. Los tratamientos conservadores son menos efectivos para reducir los síntomas de STC cuando se usan en casos avanzados moderados o severos. Los resultados quirúrgicos también son mejores para resolver los síntomas de STC y la mononeuropatía mediana focal de la muñeca subyacente cuando se realiza para los casos leves o moderados tempranos del STC (17).

Una revisión sistemática de cinco estudios que incluyeron tres ensayos clínicos aleatorizados, un control de casos y un informe de caso sugiere que las inyecciones de PRP mejoraron los síntomas de los pacientes que tenían STC leve. Esta mejora se midió utilizando el CTCB y se observaron reducciones significativas en múltiples estudios de gravedad de los síntomas. Sin embargo, este hallazgo no fue unánime y, en algunos casos, no proporcionó un beneficio mayor 
cuando se agregó a un plan de tratamiento que incluía una férula para muñeca conservadora (12). Dada la escasez de literatura publicada actualmente sobre este tema, existe la posibilidad de seguir estudiando las inyecciones de PRP para el tratamiento del STC.

Un metaanálisis que utilizó datos de siete ensayos clínicos con 270 muñecas en el grupo de tratamiento y 261 muñecas en el grupo de control, encontró mejoras estadísticamente significativas en el grupo de tratamiento con láser de bajo nivel para el agarre de la mano, la escala analógica visual y el potencial de acción del nervio sensorial 3 meses después del tratamiento del STC leve (13). Desafortunadamente, no se encontraron diferencias estadísticamente significativas en otros parámetros clínicos o estudios de conducción nerviosa y, al agrupar los datos, las variables del láser (es decir, potencia, frecuencia y longitud de onda) no siempre fueron exactamente consistentes. Aunque este metaanálisis produjo algunos resultados prometedores, se debe llevar a cabo un ensayo controlado aleatorio adicional bajo parámetros consistentes para llegar a conclusiones definitivas.

Dado que se planteó la hipótesis de que la terapia de ondas de choque dependería de la dosis, un estudio posterior probó múltiples dosis de ondas de choque. El estudio se estableció para que los pacientes fueran asignados al azar a recibir tres tratamientos, un tratamiento o ningún tratamiento. El grupo que recibió tres tratamientos durante 14 semanas mostró una reducción significativa en su puntaje CTCB con pacientes que tenían STC moderado que mostraban una reducción absoluta mayor que aquellos con STC leve (14). En resumen, se requirieron múltiples sesiones de ondas de choque para producir un resultado significativo en comparación con el placebo o los grupos de una sola sesión, lo que indica que los efectos de las ondas de choque probablemente tienen una naturaleza acumulativa.

\section{CONCLUSIONES}

El síndrome de túnel carpiano es la neuropatía periférica por compresión más comúnmente diagnosticada en todo el mundo. La afección generalmente se presenta con síntomas primarios de dolor, entumecimiento y parestesia en los primeros tres dedos, pero puede estar asociada con complicaciones que se desarrollan con el tiempo, como debilidad de la mano, disminución de las habilidades motoras finas, torpeza y atrofia visible posterior.

La mayoría de los casos de STC son idiopáticos, pero se cree que los factores de riesgo incluyen la edad y el embarazo, así como afecciones como diabetes, hipotiroidismo y artritis. Los mecanismos directos por los cuales estos factores de riesgo predisponen a los pacientes a padecer de esta patología son actualmente desconocidos.

EI STC ha sido diagnosticado tradicionalmente mediante la combinación de la historia clínica y las maniobras de exámenes físicos, como la prueba de Tinel y la prueba de Phalen, pero el advenimiento de la nueva tecnología ha generado controversia en torno al estándar de oro para el diagnóstico. Algunas escuelas creen que las sensibilidades y especificidades variables de las maniobras provocativas los hacen poco confiables y que las técnicas como las pruebas electrodiagnósticas, ultrasonido o una combinación de ambas deben ser favorecidas por su capacidad de mirar más directamente a la fisiopatología subyacente.

Los tratamientos para son variables y dependen de la gravedad del síndrome y las preferencias del paciente y el médico. El tratamiento conservador generalmente se considera una terapia de primera línea para pacientes que se presentan con STC no deficitario, pero el tratamiento generalmente se intensifica a intervención quirúrgica cuando un paciente presenta o progresa 
a déficits sensoriales o motores severos. Dada la etiología desconocida y potencialmente compleja de CTS, los estudios futuros deberían continuar explorando combinaciones de técnicas de diagnóstico existentes y potencialmente nuevas para determinar un estándar de oro universalmente aceptado para el diagnóstico.

Los médicos deben considerar el manejo del STC de manera similar a otras enfermedades epidémicas y crónicas mediante el uso de educación y controles rápidos en visitas de salud regulares. Con una disminución en el riesgo del individuo de desarrollar la enfermedad y un diagnóstico temprano usando pruebas electrodiagnósticas, con el objetivo de reducir la carga de enfermedad y su alto tratamiento médico asociado.

\section{REFERENCIAS}

1. Ashworth et al. Carpal Tunnel Syndrome. Medscape. Drugs \& Diseases. Physical Medicine and Rehabilitation. Available: https://emedicine.medscape.com/article/327330overview\#a6. 2020.

2. Mooar et al. Management of Carpal Tunnel Syndrome. J Am Acad Orthop Surg. Vol. 26(6):e128-e130. 2018.

3. Academy of Orthopaedic Physical Therapy. Carpal Tunnel Syndrome: Treating Hand Pain and Numbness. J Orthop Sports Phys Ther. Vol. 49(5):361. doi:10.2519/jospt.2019.0502. 2019.

4. Wipperman J GK. Carpal Tunnel Syndrome: Diagnosis and Management. Am Fam Physician. Vol. 94(12):993-999. 2016.

5. Sevy J VM. Carpal Tunnel Syndrome. [Updated 2020 Mar 30]. In: StatPearls [Internet]. Treasure Island (FL). Available from: https://www.ncbi.nlm.nih.gov/books/NBK448179/. 2020.

6. Masson P. Clinical Examination Versus Electrodiagnostic Assessment of Severity of Median Nerve Pathology in Carpal Tunnel Syndrome. J Clin Rheumatol. Vol. 22(1):45-46. doi:10.1097/RHU.0000000000000345. 2016.

7. Urits et al. Recent Advances in the Understanding and Management of Carpal Tunnel Syndrome: a Comprehensive Review. Curr Pain Headache Rep. Vol. 23(10):70. doi:10.1007/s11916-019-081. 2019.

8. Wang L. Guiding Treatment for Carpal Tunnel Syndrome. Phys Med Rehabil Clin N Am. Vol. 29(4):751-760. doi:10.1016/j.pmr.2018.06.009. 2018.

9. Shin E. Endoscopic Versus Open Carpal Tunnel Release. Curr Rev Musculoskelet Med. Vol. 12(4):509-514. doi:10.1007/s12178-019-09584-0. 2019.

10. Eroğlu A SE. Recurrent carpal tunnel syndrome: Evaluation and treatment of the possible causes. World J Clin Cases. Vol. 6(10):365-372. 2018.

11. Mezian K BJ. Why do local corticosteroid injections work in carpal tunnel syndrome, But not in ulnar neuropathy at the elbow? Muscle Nerve. Vol. 54(2):344. 2016.

12. Senna M. Platelet-rich plasma in treatment of patients with idiopathic carpal tunnel syndrome. Clin Rheumatol. Vol. 38(12):3643-3654. doi:10.1007/s10067-019-04719-7. 2019. 
13. Tezcan et al. Carpal Tunnel Syndrome: Evaluation of the Effects of Low-Level Laser Therapy With Ultrasound Strain Imaging. J Ultrasound Med. Vol. 38(1):113-122. doi:10.1002/jum.14669. 2019.

14. Kim J. Effect of extracorporeal shockwave therapy on carpal tunnel syndrome: A systematic review and meta-analysis of randomized controlled trials. Medicine (Baltimore). Vol. 98(33):e16870. doi:10.1097/MD.0000000000016870. 2019.

15. Gaspar et al. Sleep disturbance and response to surgical decompression in patients with carpal tunnel syndrome: a prospective randomized pilot comparison of open versus endoscopic release. Acta Biomed. Vol. 90(1):92-96. 2019.

16. Hegmann et al. Median Nerve Symptoms, Signs, and Electrodiagnostic Abnormalities Among Working Adults. J Am Acad Orthop Surg. Vol. 26(16):576-584. 2018.

17. Raizman N. AAOS Appropriate Use Criteria: Management of Carpal Tunnel Syndrome. J Am Acad Orthop Surg. Vol. 26(6):e131-e133. 2018. 